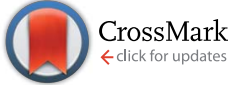

Cite this: RSC Adv., 2017, 7, 14279

Received 23rd January 2017 Accepted 27th February 2017

DOI: $10.1039 / c 7 r a 01024 a$

rsc.li/rsc-advances

\section{Influence of side chain characteristics on the aggregation-induced emission (AIE) properties of tetrasubstituted tetraphenylethylene (TPE) $\dagger$}

\author{
Samuel A. Ceballos, ${ }^{\text {ab }}$ Salvador Gil ${ }^{\mathrm{abc}}$ and Ana M. Costero*abc \\ Five new tetraphenylethylene (TPE) derivatives were synthesised and their aggregation-induced emission \\ (AIE) was evaluated in mixtures of $\mathrm{THF} /$ water and $\mathrm{MeOH} /$ water. The influence of regiochemistry and \\ length of the chains in the substituents on emissive properties was studied.
}

\section{Introduction}

Aggregation-induced emission (AIE) is a well-known phenomenon that appears in some organic molecules. ${ }^{1}$ AIE allows systems to be constructed with different properties based on aggregation properties. Hence this transduction mechanism has been used to design chemosensors for different analytes, ${ }^{2}$ electrochemical devices, ${ }^{3}$ bioprobes, ${ }^{4}$ and new fluorescent materials. ${ }^{5}$

Among the different structures likely to exhibit AIE, tetraphenylethylene (TPE) proved the most interesting. It is wellknown that tetraarylethylene is generally non-emissive in solution due to the rotations of the phenyl rings along the $=\mathrm{C}-\mathrm{Ar}$ axes and the twisting $\mathrm{C}=\mathrm{C}$ bond in the excited state, both of which serve as non-radiative relaxation channels for the excited state to decay. In the solid state or upon the addition of an antisolvent, photoluminescence of TPE derivatives can turn on. It is believed that the restriction of molecular motion $\left(\mathrm{C}_{\text {alkene }}-\mathrm{C}_{\text {arene }}\right.$ bond rotations) in solid or aggregated states is responsible for this photoluminescence activity (i.e., aggregation-induced emission, or AIE). ${ }^{6}$ The AIE properties of TPE derivatives are strongly dependent on the nature of the substituents present in the structure. ${ }^{7}$ Indeed K. Han and B.-K. Cho reported the emission properties of two luminogens that possess an identical extended tetraphenylethylene (TPE) aromatic core, but different hydrophobic properties in peripheral chains. ${ }^{8}$

In order to acquire additional information about the influence of TPE substituents on the fluorescent properties of different systems, we prepared the compounds shown in Chart 1

${ }^{a}$ Instituto Interuniversitario de Investigación de Reconocimiento Molecular $y$ Desarrollo Tecnológico (IDM), Universitat Politécnica de València, Universitat de València, Spain

${ }^{b}$ Departamento de Química Orgánica, Universidad de Valencia, Doctor Moliner 50, 46100, Burjassot, Valencia, Spain. E-mail: ana.costero@uv.es ${ }^{c}$ CIBER de Bioingeniería, Biomateriales y Nanomedicina (CIBER-BBN), Spain

$\dagger$ Electronic supplementary information (ESI) available. See DOI: 10.1039/c7ra01024a

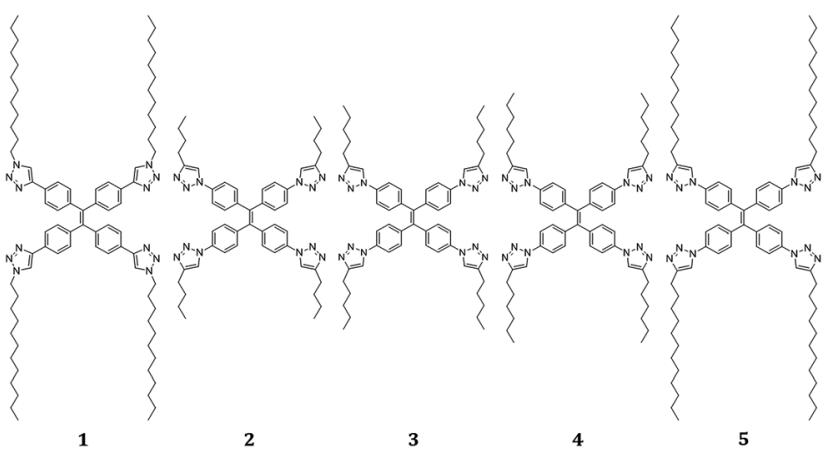

Chart 1 Synthesised TPE derivatives.
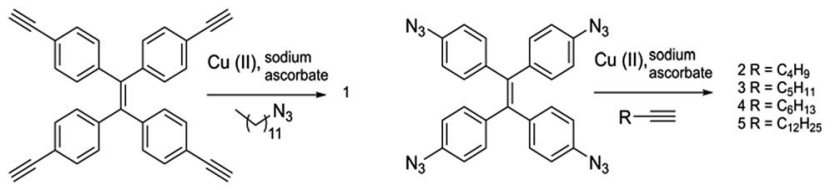

Scheme 1 Synthesis of compounds 1-5.

to know the influence that the position of the $\mathrm{N}$-atom has on the AIE properties of these compounds. The obtained results shed light on the rational design of chemosensors, or other devices, when a "click" reaction is involved in the synthetic pathway to bind a receptor moiety to the TPE used as a signalling unit.

We also studied the changes induced in AIE properties by changing the length of peripheric chains to acquire information about how hydrophobic characteristics influence their optical properties (Scheme 1).

\section{Results and discussion}

All the compounds were prepared through a "click" reaction using appropriate alkyne and azide derivatives. ${ }^{9}$ Thus compound 1 was prepared from tetrakis(4-ethynyl)tetraphenylethylene, ${ }^{\mathbf{1 0 , 1 3}}$ 
and 1-dodecylazide ${ }^{17}$ in the presence of copper(II) acetate and sodium ascorbate. ${ }^{11}$

Compounds 2-5 were prepared from the corresponding tetraazide-TPE, ${ }^{\mathbf{1 2 , 1 4 - 1 6}}$ and 1-hexyne, 1-heptyne, 1-octyne and 1-tetradecyne respectively following the same procedure.

The UV-vis spectra of compounds 2-4 showed a band at $280 \mathrm{~nm}$ and a shoulder at around $340 \mathrm{~nm}$ in the THF solutions. The UV-vis spectrum of compound $\mathbf{1}$ in the same solvent showed the main absorption band at the same wavelength (280 $\mathrm{nm}$ ) as compounds $\mathbf{2 - 4}$, but the shoulder wavelength was batochromically shifted by around $10 \mathrm{~nm}$. Compound $\mathbf{5}$, in addition to the main absorption band at 280 and the shoulder at $340 \mathrm{~nm}$, showed another shoulder around $300 \mathrm{~nm}$. The influence of solvent polarity on the UV-vis absorption bands of the studied compounds was weak (see the ESI $\dagger$ to compare the spectra in THF and $\mathrm{MeOH}$ ). After stablishing that the prepared compounds were scarce fluorescent in solid state, the fluorescence properties of the five compounds were measured first in both THF and MeOH. Compounds 2-5 were non-fluorescent in these two solvents. In contrast, compound $\mathbf{1}$ was slightly fluorescent in $\mathrm{MeOH}$, but not at all fluorescent in THF. When water was added to solutions, fluorescence for the five compounds clearly enhanced. The observed increment was dependent on both chain length and the position of the N-atom. Fig. 1 shows the steady-state fluorescence spectra of TPE derivatives $\mathbf{1}$ and $\mathbf{5}$ in $\mathrm{MeOH}$ and in the $\mathrm{MeOH} / 10 \%$ water mixture (for other $f_{\mathrm{w}}$ values, see ESI S-10†). As seen in Fig. 1, the enhanced fluorescence in the presence of water was stronger in compound 1 than in compound 5 . Thus when $f_{\mathrm{w}}$ was $10 \%$, the emissions of compounds 1 and 5 were respectively 200 and 80 times higher than the emission in pure $\mathrm{MeOH}$. Additionally, the fluorescence of compound 1 increased steadily with the proportion of water until the greatest emission was achieved in the presence of $30 \%$ water. Conversely, the emission intensity of compound 5 in $10 \%$ water was not enhanced in larger volumes of water.

Similar studies were carried out in THF with increasing amounts of water (see the ESI $\dagger$ ). Similar behaviour was observed for both compounds in this solvent, but a higher concentration of water (80-90\%) was needed to achieve the maxima emission intensity. A concentration of water above $90 \%$ gave rise to diminishing emission, as reported in the literature. ${ }^{8}$

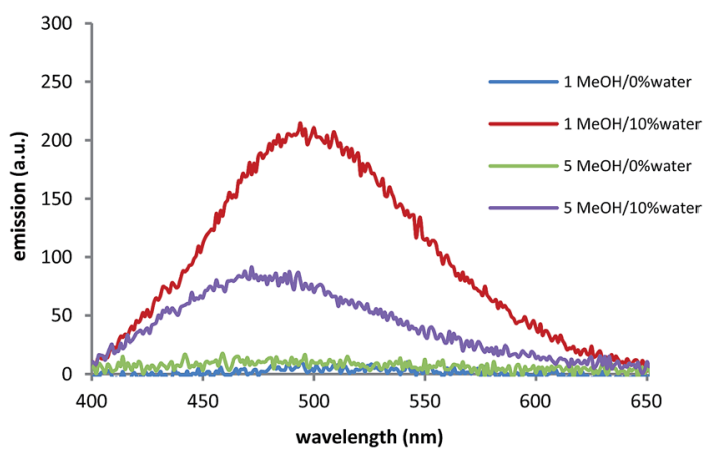

Fig. 1 The steady-state fluorescence spectra of TPE derivatives 1 and $5\left(10^{-5} \mathrm{M}\right)$ in $\mathrm{MeOH}$ and $\mathrm{MeOH} / 10 \%$ water $\left(\lambda_{\text {ex }}=350 \mathrm{~nm}\right.$, slits $2.5 \mathrm{~nm} / 5 \mathrm{~nm})$.
The comparative studies carried out in $\mathrm{MeOH}$ clearly indicated that the compound structure dramatically affected the solvent effect. Thus whereas addition of water strongly influenced the emission of compound $\mathbf{1}$, the effect on compound $\mathbf{5}$ was clearly weaker (Fig. 2). To clarify whether this behaviour was related to its solubility in $\mathrm{MeOH} /$ water, the comparative NMR spectra in the deuterated $\mathrm{MeOH}$ led to the conclusion that the solubility of both 1 and 2 was similar.

To shed some light on the reason for the higher emission of 1, a theoretic study about charge distribution and preferential conformation in both compounds was carried out (DFT calculations using basis set B3LYP/6-31G(d)). The obtained results are depicted in Fig. 3 for the corresponding model compounds used in the theoretic study. As we can see, while coplanarity is observed between the phenyl and triazol rings in compound 1 (dihedral angle of $7^{\circ}$ ), a dihedral angle of around $25^{\circ}$ is observed in compound 5. Compound $\mathbf{5}$ also shows higher polarity in the bonds of the lateral chains to the triazol ring. Atomic charges were achieved by performing a natural population analysis of them (see the ESI $\dagger$ ).

So a better packing arrangement of $\mathbf{1}$ versus $\mathbf{5}$ is expected due to both the flatter structure of $\mathbf{1}$ and the greater charge distribution in 5 . The change in the polarity of the solvent from adding water influenced compound $\mathbf{1}$ more than compound $\mathbf{5}$. Consequently, the maximum fluorescence enhancement after adding water was achieved with $f_{\mathrm{w}} 10 \%$ for 5 , and monotonously increased to $f_{\mathrm{w}}=30 \%$ for compound 1 .

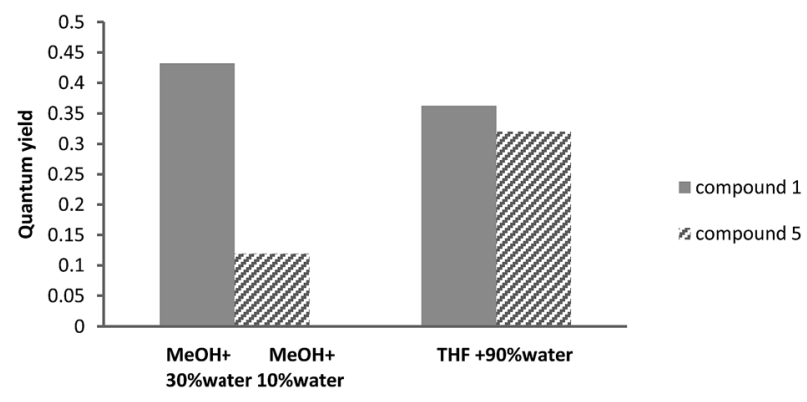

Fig. 2 Influence of compound structure on the solvent effect. The $f_{\mathrm{w}}$ value to determine quantum yield corresponded to maximum enhancement.

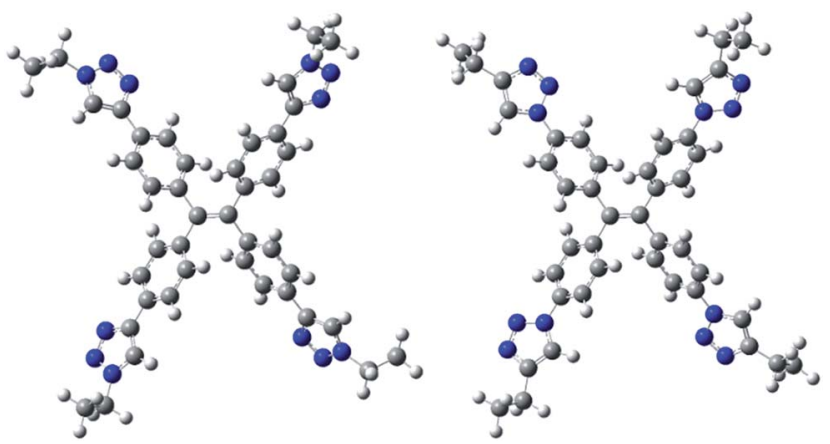

Fig. 3 The conformation calculated for the model compounds (left) for 1 and (right) for 5 . 
The studies carried out in THF demonstrated that the solubility of both compounds in this solvent was greater than in $\mathrm{MeOH}$. For this reason, larger volumes of water were needed to induce aggregation and, consequently, to enhance fluorescence.

We also observed that no changes in emission band wavelength were observed in $\mathrm{MeOH}$ after adding increasing amounts of water. However, a hypsochromic shift of $40 \mathrm{~nm}$ and $60 \mathrm{~nm}$ was observed in THF for compounds 1 and 5, respectively, when $f_{\mathrm{w}}$ changed from $20 \%$ to $80 \%$.

The SEM images of compounds 1 and 5 in $\mathrm{MeOH}-40 \%$ water are shown in Fig. 4. The comparison of the SEM images of both compounds shows that both have a similar morphology, and the only difference was the size of spheres, which are larger for compound 1 than for compound 5. Conversely, a different morphology is observed in THF-80\% water, which is laminar with a clear piling tendency. As revealed by the fluorescence microscopic images (Fig. 5), the morphology of particles is consistent with the SEM images.

Finally, a comparative study with compounds 2-5 in $\mathrm{MeOH}$ demonstrated that the minimum $f_{\mathrm{w}}$ needed to induce enhanced emission intensity lowered as chain length increased. So whereas around $60 \%$ water was required for compound $2,30 \%$, $30 \%$ and $10 \%$ were needed for 3, 4 and 5, respectively. Larger amounts of water did not induce substantial increments in fluorescence in any case.

These results (Fig. 6) clearly indicate that the longer the hydrophobic chain, the lower compound solubility becomes in aqueous mixtures. This is consistent with its solubility in deuterated methanol, as determined by NMR studies.

(a)

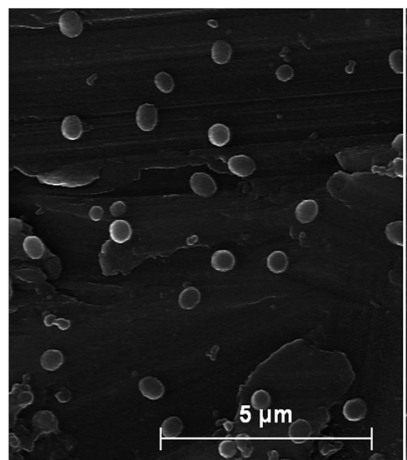

(c)

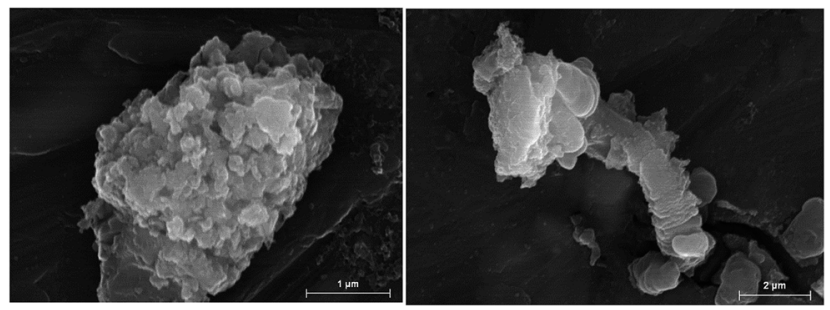

Fig. 4 SEM images of the aggregate morphologies in $\mathrm{MeOH}-40 \%$ water of (a) 1 and (b) 5 . Morphologies in THF-80\% water of (c) 1 and (d) 5 .

When the four compounds were studied in the same solvent mixture (MeOH/water 10/80) (Fig. 7), maximum fluorescence enhancement was observed for compound $\mathbf{3}$, followed by 4 . Conversely, shorter aliphatic chains (compounds 2 and 5)

(a)

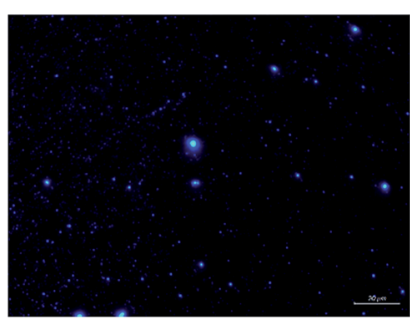

(c)

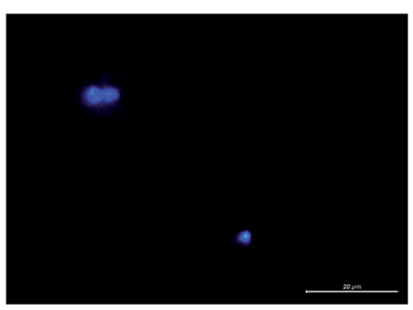

Fig. 5 Optical fluorescence microscopy of (a) 1 and (b) 5 in $\mathrm{MeOH}$ $40 \%$ water and (c) 1 and (d) 5 in THF-80\% water.

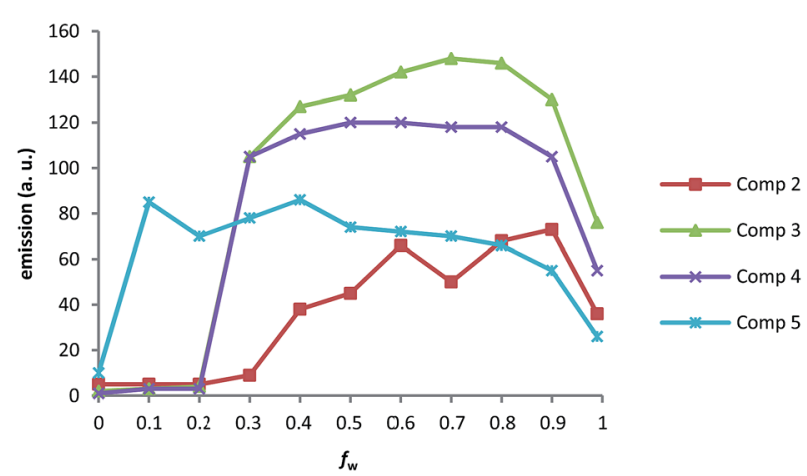

Fig. 6 The main band intensity of compounds $2-5$ in the $10^{-5} \mathrm{M}$ $\mathrm{MeOH} /$ water mixtures.

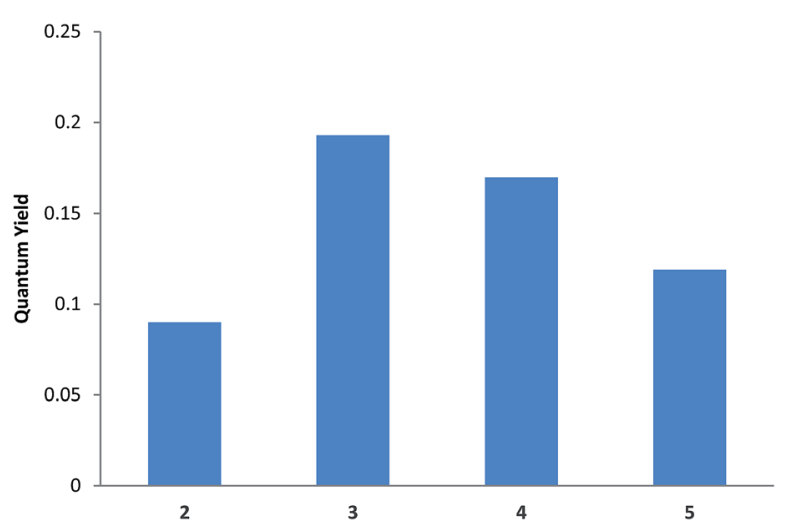

Fig. 7 Quantum yields of compounds $2-5$ in $\mathrm{MeOH} / 80 \%$ water. 
exhibited lower quantum yields. Similar behaviour was observed when THF-water was used as the solvent system (see the ESI $\dagger$ ).

\section{Conclusions}

Compounds 2-5 are easy to prepare using the corresponding "click" reactions. The regiochemistry of the substituent strongly influences the effect of solvent mixtures. This behaviour may be related to the packing arrangement of the compounds in the used solvent. Theoretical studies have demonstrated that whereas coplanarity between phenyl and triazol rings is observed in compound $\mathbf{1}$ (dihedral angle $7^{\circ}$ ), a dihedral angle of around $25^{\circ}$ is observed in compound 5. Despite the similar solubility in the $\mathrm{MeOH}$ of $\mathbf{1}$ and $\mathbf{5}$, the former is more emissive and more sensitive to changes in the solvent's polarity by adding water. When comparing compounds 2-5, the obtained results clearly indicate that the longer the hydrophobic chain, the lower the solubility of the compound in $\mathrm{MeOH}$, which gives rise to different responses in mixtures $\mathrm{THF} /$ water and $\mathrm{MeOH} /$ water. Finally, an optimum length chain exists to accomplish greater fluorescence enhancement.

\section{Acknowledgements}

We thank the Spanish Government, FEDER funds (MAT201564139-C4-1-R and MAT2015-64139-C4-4-R) and the Generalitat Valenciana (PROMETEOII/2014/047) for support. SCSIE (Universidad de Valencia) is gratefully acknowledged for all the equipment employed. We thank Mar Rios for her help in the theoretic calculations.

\section{Notes and references}

1 Y. Hong, J. W. Y. Lam and B. Z. Tang, Chem. Soc. Rev., 2011, 40, 5361.

2 J. Bian, L. Xu, H. Wang and S. Feng, Phys. Chem. Chem. Phys., 2015, 17, 32472; L. Peng, Z. Zhou, R. Wei, K. Li, P. Song and A. Tong, Dyes Pigm., 2014, 108, 24; L. Liu, G. Zhang, J. Xiang, D. Zhang and D. Zhu, Org. Lett., 2008, 10, 4581; F. Sun, G. Zhang, D. Zhang, L. Xue and H. Jiang, Org. Lett., 2011, 13, 6378; Y. Zhang, D. Li, Y. Li and J. Yu, Chem. Sci., 2014, 5, 2710; W. Dong, T. Fei, A. Palma-Cando and U. Scherf,
Polym. Chem., 2014, 5, 4048; W. Dong, J. Pina, Y. Pan, E. Preis, J. S. Seixas de Melo and U. Scherf, Polymer, 2015, 76, 173.

3 S. Mi, J. Wu, J. Liu, Z. Xu, X. Wu, G. Luo, J. Zheng and C. Xu, ACS Appl. Mater. Interfaces, 2015, 7, 27511.

4 D. Ding, K. Li, B. Liu and B. Z. Tang, Acc. Chem. Res., 2013, 46, 2441.

5 X. Zhang, X. Zhang, B. Yang, S. Wang, M. Liu, Y. Zhang, L. Tao and Y. Wei, RSC Adv., 2013, 3, 9633; G. G. Shan, H. B. Li, H. Z. Sun, D. X. Zhu, H. T. Cao and Z. M. Su, J. Mater. Chem. C, 2013, 1, 1440.

6 Y. Hong, L. W. Y. Lam and B. Z. Tang, Chem. Commun., 2009, 4332.

7 H. Tong, Y. Hong, Y. Dong, M. Häußler, J. W. Lam, Z. Li, Z. Guo, Z. Guo and B. Z. Tang, Chem. Commun., 2006, 3705; W. Wang, T. Lin, M. Wang, T. X. Liu, L. Ren, D. Chen and S. Huang, J. Phys. Chem. B, 2010, 114, 5983; D. Jana, S. Boxi, P. P. Parui and B. K. Ghorai, Org. Biomol. Chem., 2015, 13, 10663; H. Imoto, K. Nohmi, K. Kizaki, S. Watase, K. Matsukawa, S. Yamamoto, M. Mitsuishi and K. Naka, RSC Adv., 2015, 5, 94344; Y. Dong, W. Wang, C. Zhong, J. Shi, B. Tong, X. Feng, J. Zhi and Y. Dong, Tetrahedron Lett., 2014, 55, 1496.

8 K. Han and B. K. Cho, RSC Adv., 2015, 5, 9510; S. B. Han, H. J. Kim, D. Jung, J. Kim, B. K. Cho and S. Cho, J. Phys. Chem. C, 2015, 119, 16223.

9 J. E. Hein and V. V. Fokin, Chem. Soc. Rev., 2010, 39, 1302. 10 V. S. Vyas and R. Rathore, Chem. Commun., 2010, 46, 1065.

11 A. M. Deobald, L. R. S. Camargo, D. Alves, J. ZukermanSchpector, A. G. Corrêa and M. W. Paixão, Synthesis, 2011, 4003.

12 S. Xu, X. Zhuang, X. Pan, Z. Zhang, L. Duan, Y. Liu, L. Zhang, X. Ren and K. Ding, J. Med. Chem., 2013, 56, 4631.

13 V. S. Vyas, M. Banerjee and R. Rathore, Tetrahedron Lett., 2009, 50, 6159.

14 J. H. Gorvin, J. Chem. Soc., 1959, 678.

15 A. Nose and T. Kudo, Chem. Pharm. Bull., 1981, 29, 1159.

16 S. Xu, X. Zhuang, X. Pan, Z. Zhang, L. Duan, Y. Liu, L. Zhang, X. Ren and K. J. Ding, J. Med. Chem., 2013, 56, 4631.

17 M. Sohail, R. De Marco, M. Pawlak, M. Zdeňka Jarolímová, E. Bakker, N. He, R. M. Latonen, T. Lindfors and J. Bobacka, Langmuir, 2015, 31, 10599. 